\title{
Application of botulinum toxin in maxillofacial field: Part II. Wrinkle, intraoral ulcer, and cranio-maxillofacial pain
}

\author{
Kyung-Hwan Kwon ${ }^{*}$ D, Kyung Su Shin, Sung Hee Yeon and Dae Gun Kwon
}

\begin{abstract}
Botulinum toxin (BTX) is used in various ways such as temporarily resolving muscular problems in musculoskeletal temporomandibular disorders, inducing a decrease in bruxism through a change in muscular patterns in a patient's bruxism, and solving problems in patients with tension headache. And also, BTX is widely used in cosmetic applications for the treatment of facial wrinkles after local injection, but conditions such as temporomandibular joint disorders, headache, and neuropathic facial pain could be treated with this drug. In this report, we will discuss the clinical use of BTX for facial wrinkle, intraoral ulcer, and cranio-maxillofacial pain with previous studies and share our case.
\end{abstract}

Keywords: Botulinum toxin, Clinical application, Maxillofacial field, Wrinkle, Oral ulcer, Maxillofacial pain

\section{Background}

In oral maxillofacial surgery, the frequency of use of botulinum toxin is increasing rapidly. Particularly, it is used not only for symptomatic treatment for a disease but also used as a method of causative treatment approach. Botulinum toxin is used in various ways such as temporarily resolving muscular problems in musculoskeletal temporomandibular disorders, inducing a decrease in bruxism through a change in muscular patterns in a patient's bruxism, and solving problems in patients with tension headache. It is used not only for treating these diseases but also for the cosmetic purpose to smoothen wrinkles caused by the muscle of expression of the face [1].

\section{Clinical use for wrinkle treatment}

When using botulinum toxin in various treatments, the clinician should be thorough with the concepts and knowledge of anatomical structures. It is true that the importance of knowledge on anatomical structures to reduce side effects and increase efficacy and ability to cope with each event keeps increasing. As botulinum toxin can be used for various treatments, accordingly, it is also

\footnotetext{
* Correspondence: kkhoms@hanmail.net

Department of Oral and Maxillofacial Surgery, College of Dentistry, Wonkwang University, Iksan, South Korea
}

important to acquire knowledge on anatomical structures for various purposes [1-5].

\section{Anatomy of facial expression muscles}

The superficial musculoaponeurotic system (SMAS) layer is an important anatomical structure when acquiring anatomical knowledge for muscles of expression. When we observe the skin of the face and muscle layers beneath the skin, the head, and the neck, SMAS is connected to the dermis at one end, and the other end is connected to fascia of facial muscles. Superiorly, it is connected to temporalis muscles and frontalis muscles, anteriorly connected to orbicularis oculi, inferiorly connected to platysma, and posteriorly connected to trapezius muscles. Description of the SMAS layer is shown schematically in Fig. 1.

The SMAS layer is also used in facelift surgery, which is a procedure to lift the SMAS layer posteroinferiorly and flatten the facial wrinkles $[6,7]$.

The frontalis muscle has no bony attachments since it originates from galea aponeurotica and inserts into the skin of the eyebrows (Fig. 2). Its function is to lift the skin around the nasal root, making wrinkles on the forehead. It is supplied by a temporal branch of the facial nerve and supraorbital and supratrochlear artery. It is responsible for forehead crease, and botulinum toxin 


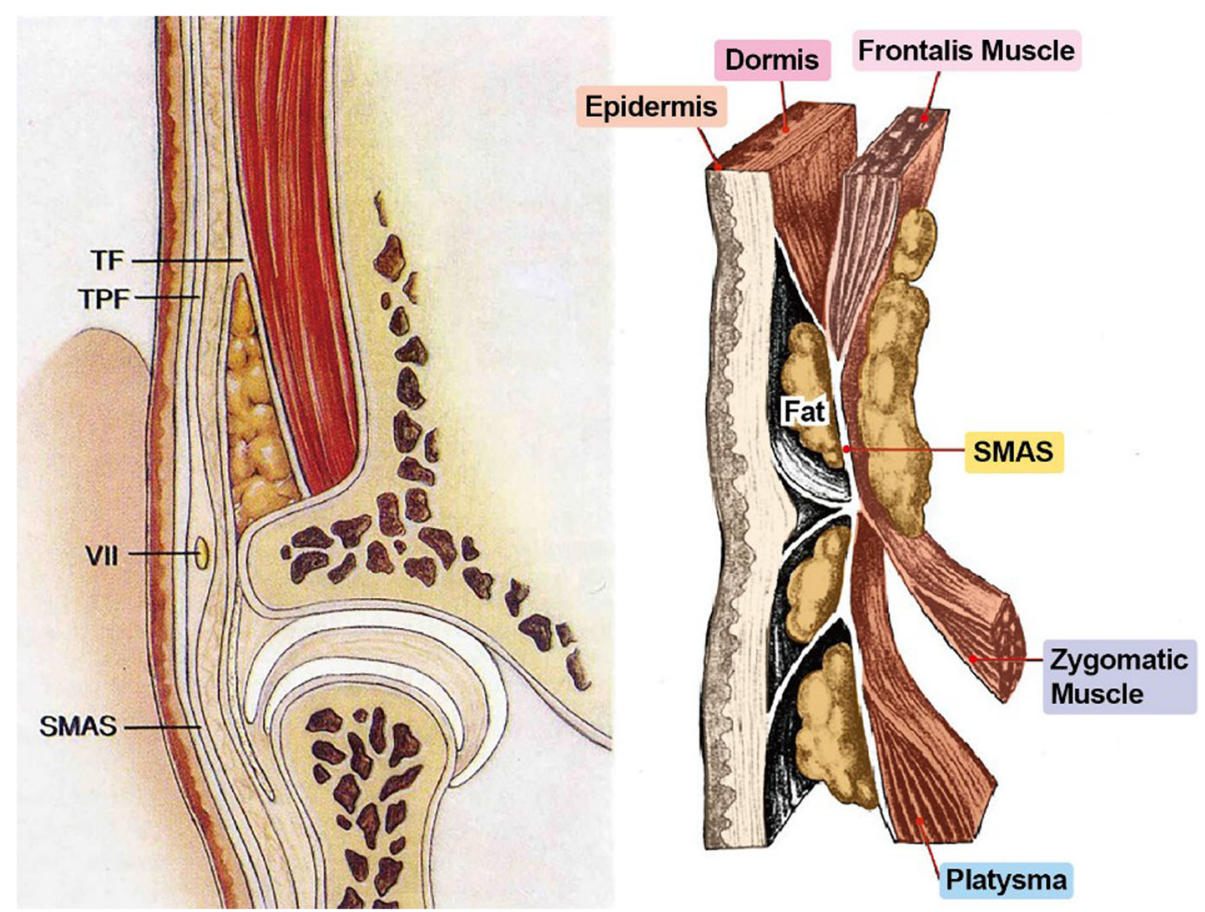

Fig. 1 Schematic representation of superficial musculoaponeurotic system (SMAS)

injection can change the pattern of creases through flaccid paralysis of contraction of muscles. Wrinkles will reappear after 6 months, but in a different pattern than that of the past.

Orbicularis oculi muscles is connected to the nasal part of the frontal bone and frontosphenoidal process by medial palpebral ligament and lateral palpebral ligament, respectively. It is responsible for closing of the eyes.

Corrugator supercilii is located beneath the frontalis and orbicularis oculi, and it draws the eyebrows medially downward. It is supplied by the temporal branch of the facial nerve and supreatrochlear artery. It is used as the principal target muscle during botulinum toxin injection as it is regarded as the muscle causing glabellar lines.

Risorius originates from the fascia of the masseter muscle, proceeds horizontally forward, and inserts into the skin at the corner of the mouth. Its function is to pull the corner of the mouth sideward to produce a smile. It is innervated by the buccal branch of the facial nerve and supplied by the transverse facial artery and facial artery. Injecting botulinum toxin into the orbiculis oris affects the risorius muscle leading to drooping of the lips [8].

\section{Treatment of glabellar lines}

The proceus muscles, corrugator supercilii muscles, and depressor supercilii muscles are involved in the formation of glabellar lines. A good treatment effect is expected for wrinkles formed during the facial movement, but for deep wrinkles, using collagen matrix or injecting fillers may be recommended. In general, the effect is to last for 3-5 months. Repeated injections may not completely remove wrinkles, but once the wrinkles start to deform, you can observe the wrinkles disappearing. With every 6 months of botulinum injection, the wrinkles begin to deform, and if this persists, they will be transformed into different forms from the existing wrinkles $[6,9,10]$.

It is important to target the corrugator muscle while injecting botulinum toxin. In our case, we mix BTXA (Hanall pharmaceuticals) with $2.5 \mathrm{cc}$ saline to make 100 units, then make 4 unit per $0.1 \mathrm{cc}$ and then inject 4 units per injection point (dosage required for each part is shown in Fig. 3). We use a $60^{\circ}$ angle and advance the needle until the bone is gently contacted. (It is different for each operator. Some operator use less than $45^{\circ}$ and some use $90^{\circ}$. Choosing the degree angle is a matter of operator's preference. Even though we randomly set the criteria for choosing the degree angle, as a matter of fact, there is a difference in the extent of diffusion depending on the degree angle.) After inserting the needle and checking the exact area, inject slightly upward direction. To reduce the effect on glabellar lines of orbicularis oculi and to cause complete paralysis of the corrugator muscle, inject $1 \mathrm{~cm}$ away from the orbital bony rim at the midpupillary line of the eyes. When we classify the patients based on the types of wrinkles, i.e., shallow lines, deep lines stretchable with hands, and permanent deep creases, you do not have to adjust the dose of botulinum toxin for shallow lines, but if 


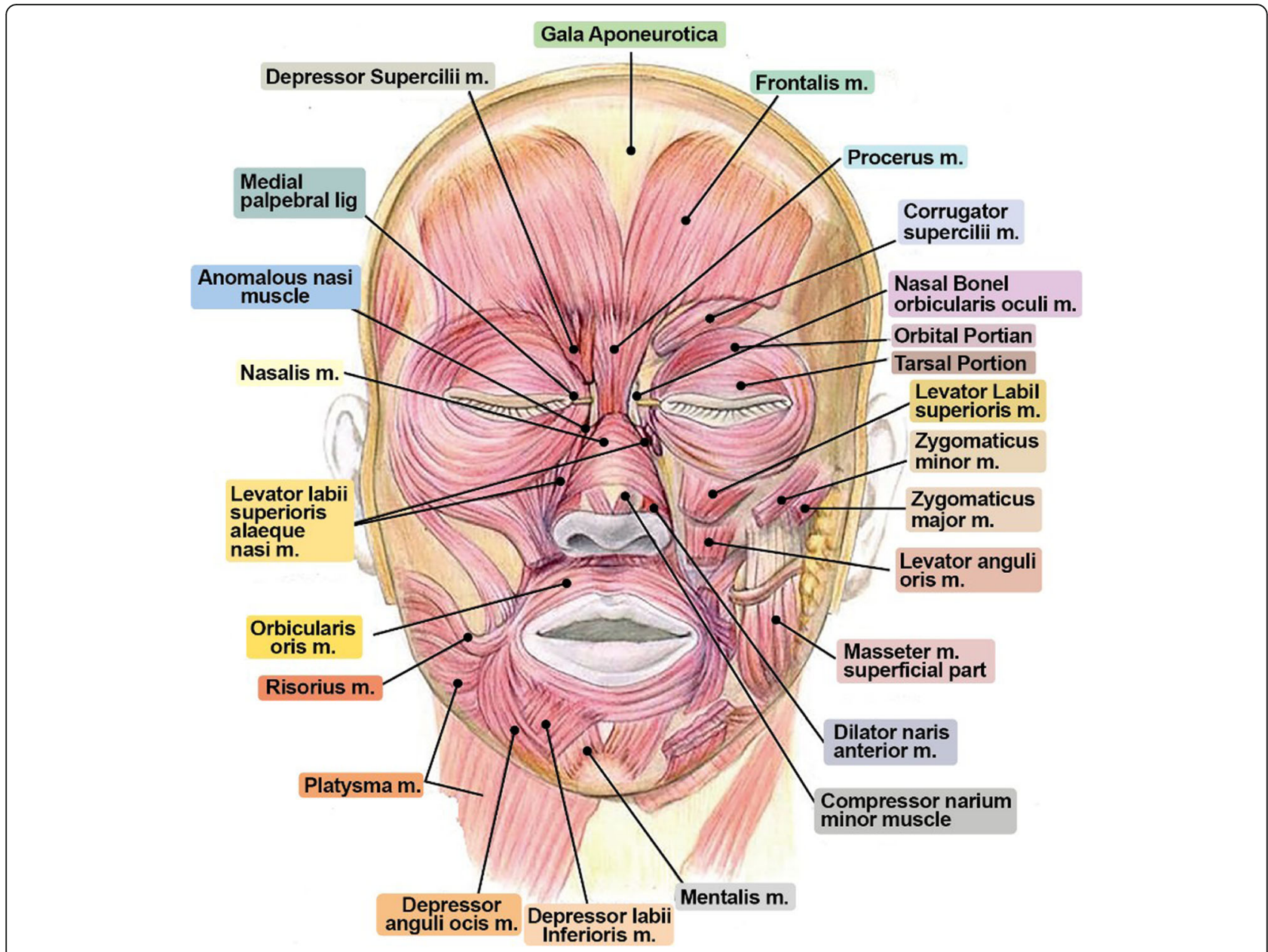

Fig. 2 It is very important to understand the origin and insertion of expression muscles of the face in wrinkle treatment. Especially, one should carefully observe the origin and insertion of frontalis muscles, corrugator supercilii m., procerus m., orbicularis oculi m., and orbicularis oris $\mathrm{m}$.

there is a case where the patient does not want a big change in his impression, you may reduce the dose as required. Inject with $50 \%$ dose into the insufficient area after a week or two for deep lines that are stretchable with hand $[11,12]$.

Lastly, you may consider using fillers after injecting with $50 \%$ of the dose into the insufficient area after a week or two for the case of permanent deep creases. The patient should be aware of additional use of fillers.

Carruthers had reported that he had given additional injections to paralyze the orbicularis on the inner side of the corrugator muscle, located $1 \mathrm{~cm}$ above the orbital bony rim. This is why we must pre-determine the location to give an additional injection into the deficient area in a week to 2 weeks [11].

While there are not many side effects from its use, upper eyelid ptosis may be the worst side effect. It is caused by partial paralysis of the levator palpebrae superioris that resolves after 2-4 weeks. To minimize side effects, it is important not to touch or press the injection site to prevent excessive diffusion of botulinum toxin around the eyes. Also, the patient should not lie down for $2-3 \mathrm{~h}$ right after the injection. The operator may reduce the dose as much as possible and must keep in mind that he would not inject botulinum toxin into the area below the eyebrows exterior to the internal canthus $[9,10]$

Treatment effect of glabellar lines with botulinum toxin will appear 3-4 days after the injection, and you can expect the significant effect only after 10 days of injection. Generally, after a week or two, you should decide whether the additional injection is required.

\section{Treatment of forehead wrinkles}

Unlike Westerners, Asians, especially Koreans, have broader a frontalis muscle that extends to the edge of the forehead rather than restricted to the center of the forehead. Therefore, it is necessary to inject the whole area including the lateral portion of the forehead. If we follow Western guidelines (injecting only in the center of the forehead), it will increase the incidence of a 


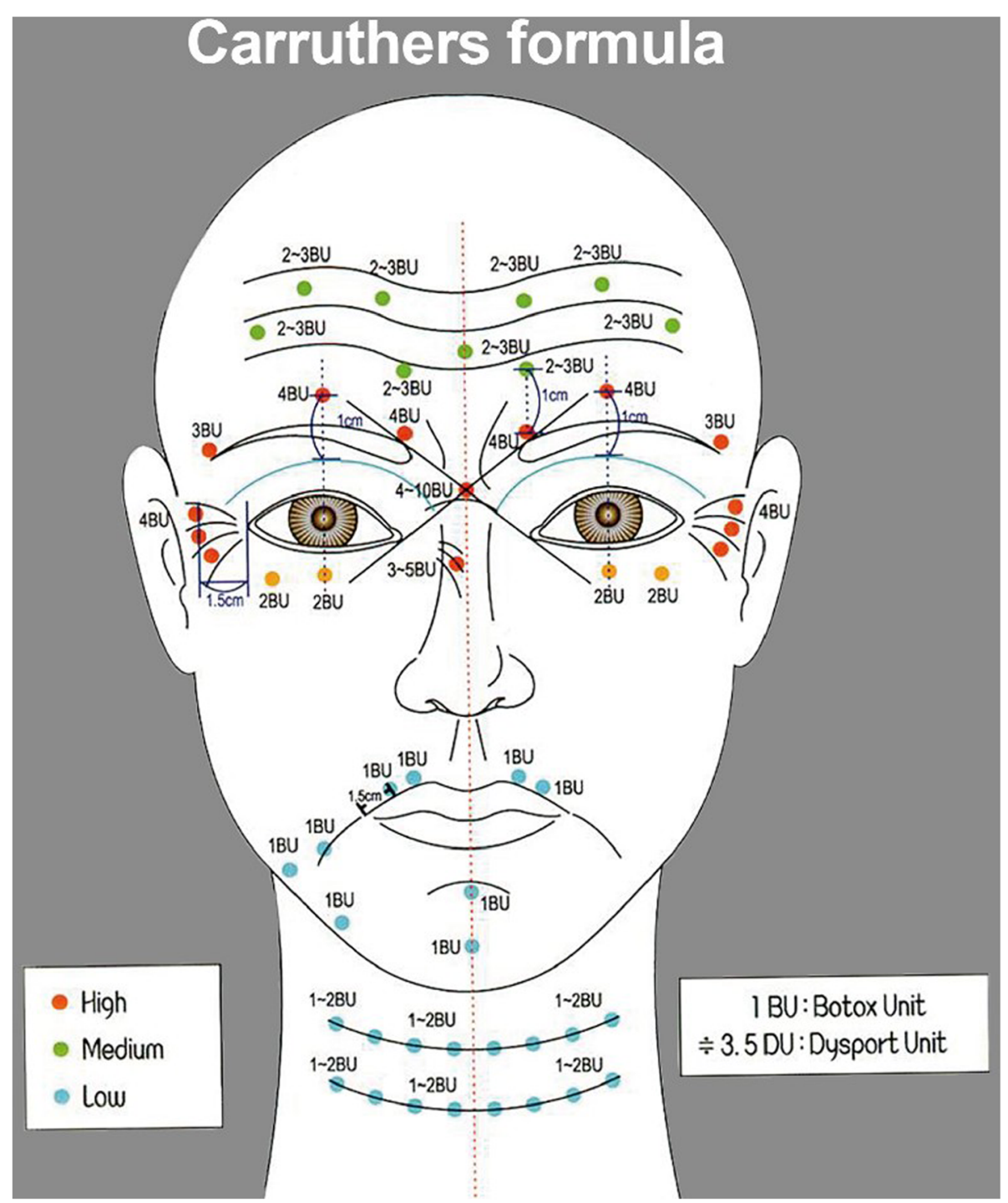

Fig. 3 A protocol used for forehead creases and eye wrinkles. The standard dose was determined based on BTXA. (formula by Carruthers)

condition called samurai eyebrows (V-shaped eyebrows). This is because the contractibility of the frontalis muscle remains which brings imbalance to the facial harmony, which eventually leads to the lifting of the eyebrows. In my early days, I also had such a case where I unintentionally caused samurai eyebrows by not injecting into the temporal muscles, but there are few such cases nowadays. The fact that large frontal bellies have no attachment point to the bone, and more of the muscles are distributed on the temporal side rather than the central area, is an important thing to remember while injecting botulinum toxin [13].

Inject one or two lines of injecting points with a distance of 1.5 to $2.5 \mathrm{~cm}$ depending on the width of the forehead, the depth of the wrinkles, the number of wrinkles, and the distribution of the wrinkles. According to Khawaja, and
Hassan Abbas et al., toxins spread $2.5-3 \mathrm{~cm}$ in all directions from the injection point in the case of the front forehead, and the effect spreads uniformly. When we inject with $2 \mathrm{U}$ ( $0.05 \mathrm{cc}$ per injection point), the range of botulinum toxin diffusion is generally about $1.5 \mathrm{~cm}$ in radius, $3 \mathrm{~cm}$ in diameter (Fig. 4).

In Koreans, injecting 4-10 points would be sufficient to treat both glabellar lines and forehead wrinkles. Imagine there is a vertical line drawn on the outer boundary of the eyebrows. Inject lateral to the line, both left and right sides of the face. Inject 4-6 points symmetrically with a distance of $2 \mathrm{~cm}$ into the horizontal line drawn $2.5 \mathrm{~cm}$ above the orbital rim. If there are wrinkles in the upper part of the central region, give one or two additional injections. Use $2 \mathrm{U}$ per each injection (Fig. 5). 


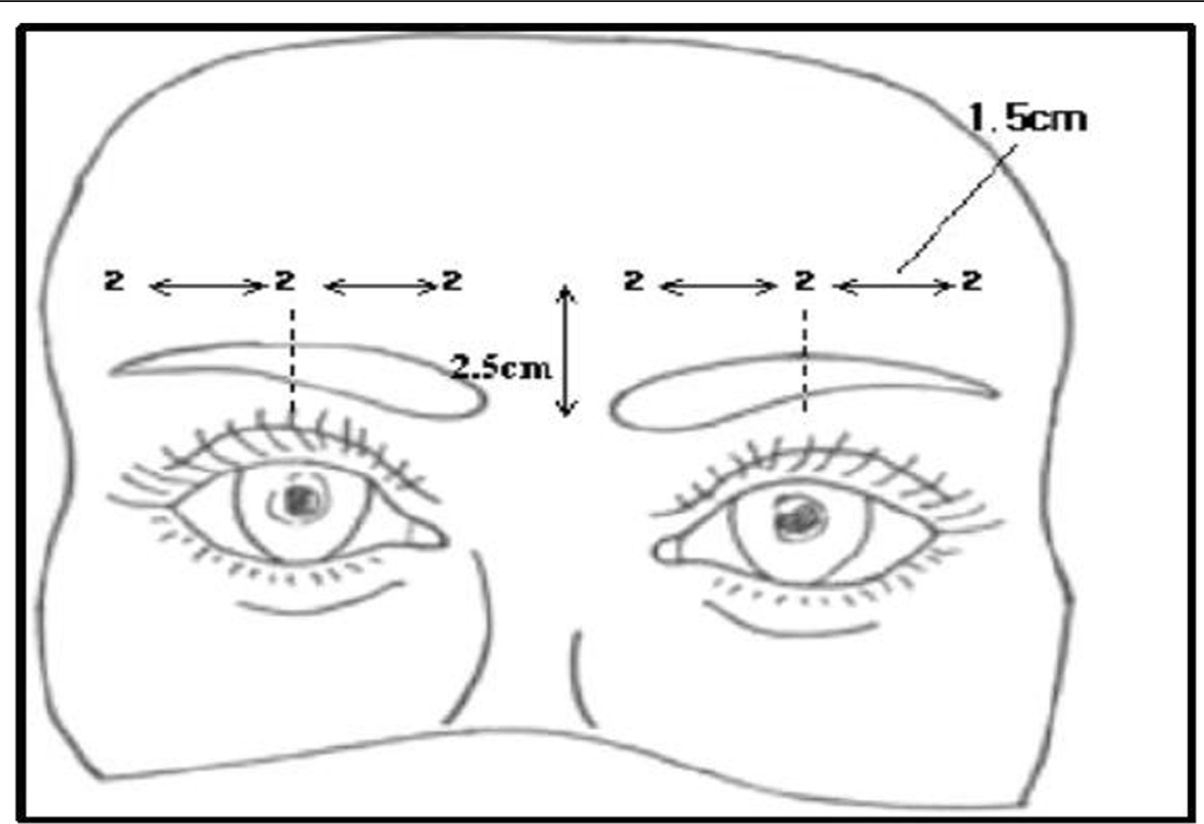

Fig. 4 Schematic representation of injection points for forehead wrinkles. Injection point

Side effects include eyebrow ptosis, upper eyelid swelling, and samurai's eyebrows. If you comply with the standard precautions, there won, give one or two a $[9,10]$.

Figure 5 shows 2 weeks after the injection of BTXA into the glabellar lines. The patient tries hard to make wrinkles by raising her eyebrows. For deep wrinkles that one can spread by hand, the effect of botulinum toxin will be the most prominent.

\section{Treatment of eye wrinkles (angulus oculi lateralis} wrinkles)

Eye wrinkles are closely related to the aging progress. After the 30s, skin elasticity decreases and smile wrinkles start to appear. As the fibroelastosis continue to progress, the wrinkles resembling crow's feet tend to develop and this eventually is recognized as a sign of aging. The so-called crow's feet wrinkles are considered to be

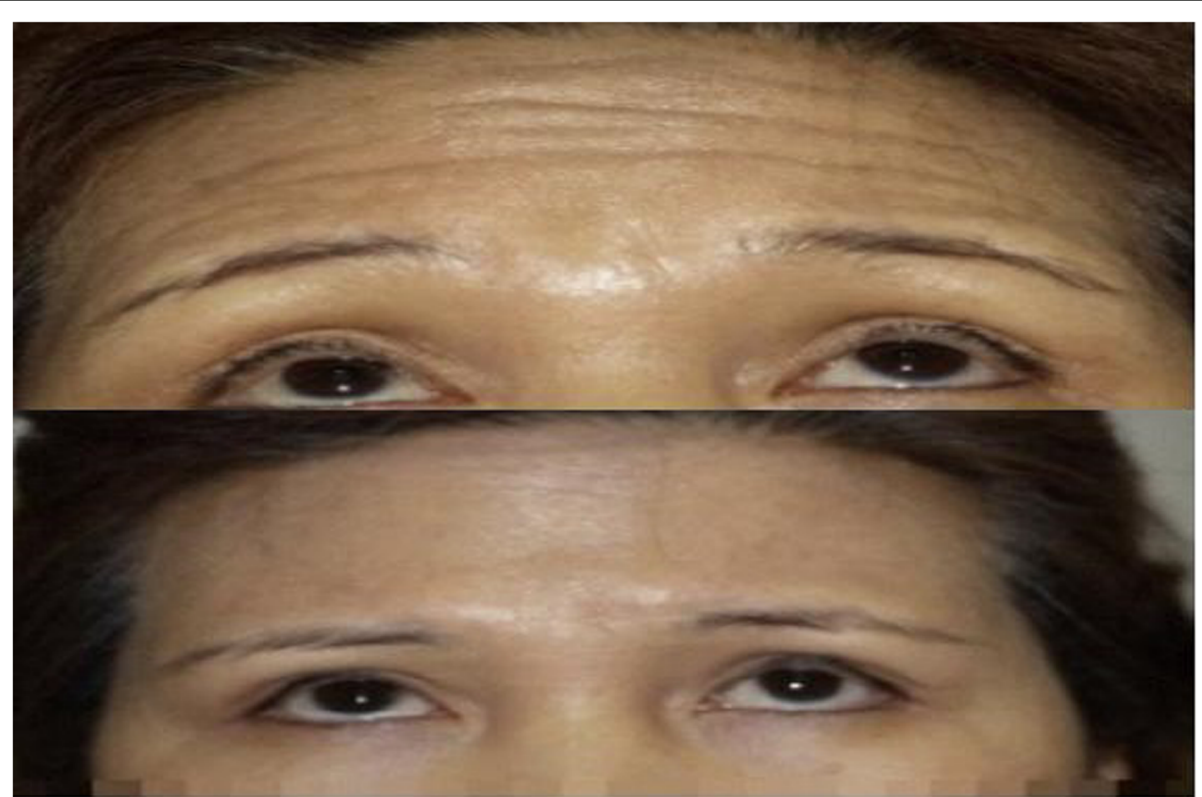

Fig. 5 (Up) Initial diagnosis, forehead wrinkles are observed. (Down) 2 weeks after BTXA injection 
caused by the intrinsic aging of the skin and the photoaging by ultraviolet rays as well as the worsening smile lines due to the chronic and excessive contraction of the orbicularis oculi and the fibrous thickening of the orbicularis oculi muscle $[14,15]$.

It is a basic principle to inject a high-concentrationsmall-dose injection of botulinum toxin for the treatment of eye wrinkles. This is a precise, long-lasting method, and has fewer side effects than lowconcentration-high-dose injection. Normally, $0.1 \mathrm{cc}$ injection allows effective injection in $4 \mathrm{U}$ increments after dilution with $2.5 \mathrm{cc}$ of distilled water or saline. In general, it is essential to inject $1 \mathrm{~cm}$ per point $1.5 \mathrm{~cm}$ away from where the orbital bone is felt (Fig. 6) [11, 12].

It is advised to inject at a $45^{\circ}$ angle because the skin is thin. After injection, gently rub the area toward the opposite direction of the orbit to evenly spread the toxin. As it is easy to bruise, it is also a good way to reduce the risk of bruising by shrinking blood vessels while reducing pain with local anesthetics. Make sure the patient does not lie down for 2 to $3 \mathrm{~h}$ after the procedure as the injection may flow to the nose or may spread to the unwanted area (Fig. 7).

\section{Clinical use for intraoral ulcer}

Treatment of oral ulcerative lesion using botulinum toxin It is reported to be effective in the treatment of oral ulcers in some literature. However, more studies on clear mechanisms and efficacy seem necessary. It has been reported to be effective in anal fissure. An anal fissure is a lacerated wound of the anal canal from the anal verge to the dentate line. In the acute phase, a simple laceration becomes chronic and ulcers are formed. The cause was unknown in the past $[16,17]$. Local ischemia at the anal fissure site plays an important role in the cause of disease. In such a case, when the internal anal sphincter is injected with botulinum toxin, it is known that it relaxes the muscles, thereby increasing blood circulation treating necrosis or chronic ulcers [5].

Occurrence of oral ulcer is thought to be due to various reasons, one of which is an autoimmune disease, whereas some people think that it occurs as a result of activation of the dormant virus in the peripheral nerves during the period of trauma, endocrine disorder, menstruation, and allergy, where the immune system is significantly lowered [18].

Formation of oral ulcer is based on the hypothesis that the intraoral ulcer progress from a traumatic ulcer to a chronic ulcer due to the friction between the tooth and mucosa due to the interaction between the orbiculis oris muscle and buccinator muscle. Moreover, it is also considered that viral infection in the form of opportunistic infections is additionally involved. Therefore, along with the botulinum toxin treatment, treatment of intra-oral ulcers should be considered by eliminating certain causes and improving intra-oral environments [19].

Inject only into the mucosa and not the surrounding muscles. Generally, inject 0.5 units $(2.0 \mathrm{cc}$ of distilled water or normal saline mixed with 100 units of BTXA, use $0.01 \mathrm{cc}$ ) with the idea of injecting (infiltration) into the boundary of the ulcer about $2 \mathrm{~mm}$ away

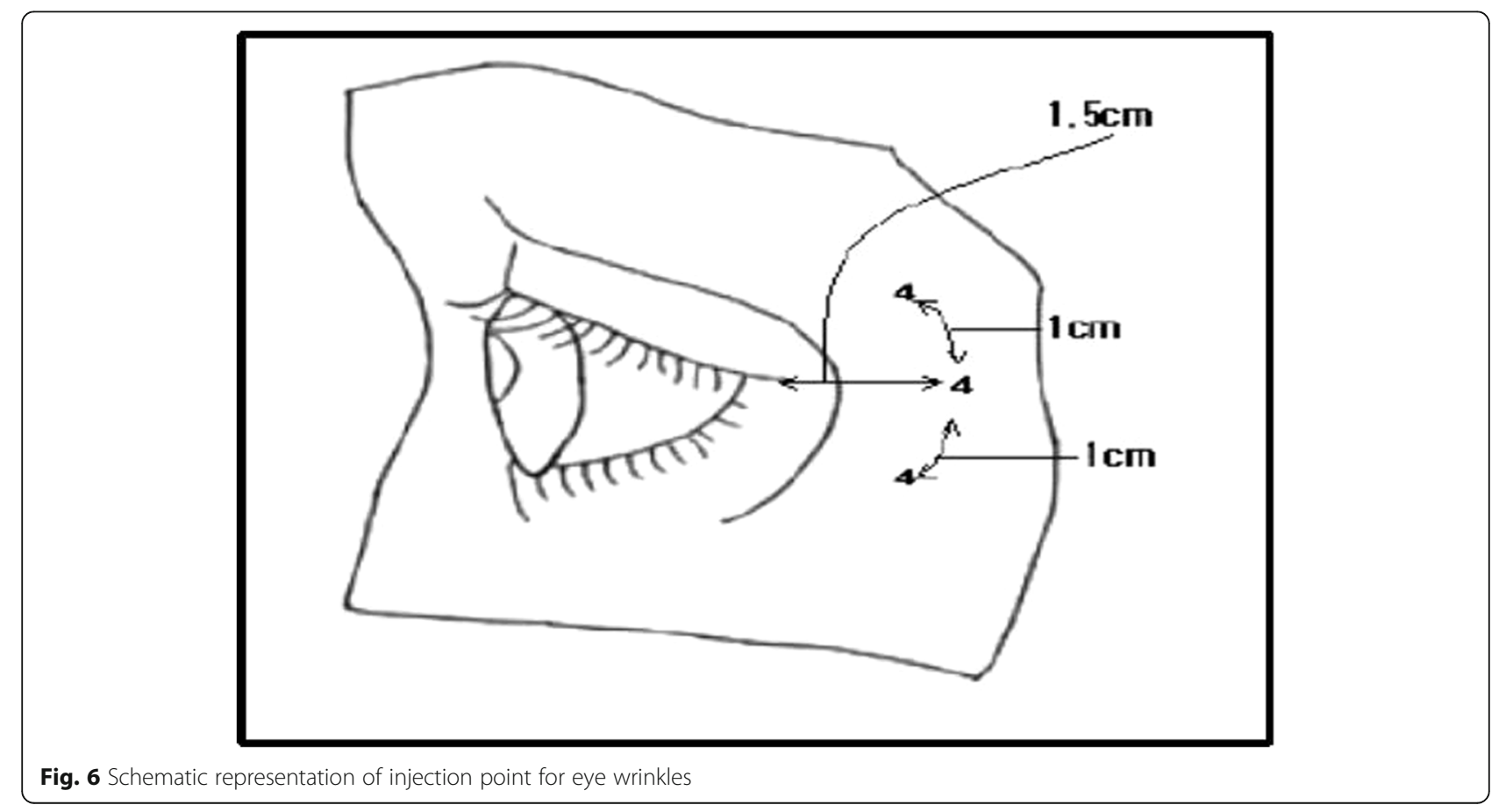




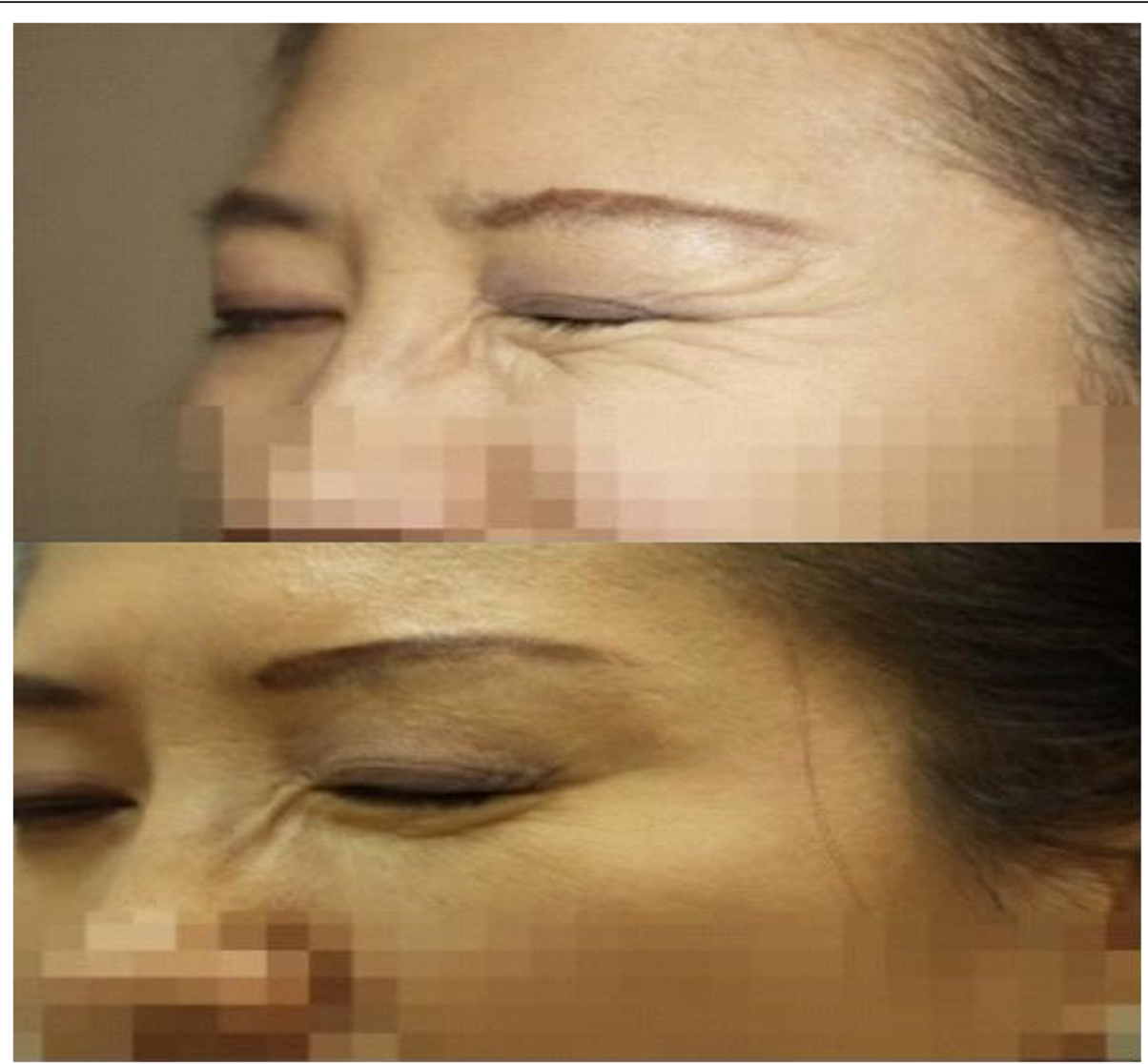

Fig. 7 Treatment of eye wrinkles. After a week of BTXA injection, the patient is not able to make eye wrinkles even if she tries to do. (Up) Patient at initial diagnosis. (Down) 1 week after BTXA injection

from the center of the ulcer. Inject one point per each ulcer.

Figure 8 shows a case of a treatment of oral ulcer by botulinum toxin in our hospital. A patient suffered from oral ulcer persisting even though the patient visited and received treatment from the Department of Oral Medicine and the Department of Periodontology. On biopsy, the ulcerative lesion was found to be erosive lichen planus, and when we injected 0.5 unit of BTXA into the mucosa surrounding the lesion and at the center of the lesion, it relieved the pain and significantly reduced the size of the ulcer.

\section{Clinical use for cranio-maxillofacial pain}

One of the most common symptoms of craniomaxillo-facial pain is headache. Headache is the most common distressing symptoms regardless of the pathophysiological mechanism, morbidity rate, and causes. In literature, headache is described as one of the 10 most common symptoms in clinical medicine, and $8 \%$ of patients who visits the hospital complains of a headache [20]. It is no exaggeration to say that most of dental clinic in-patients complain of headache when we distinguish the related pain and referred pain. The principle of treating headache is the removal of the source of pain. Even if the intrinsic structural lesion does not appear in most patients who complain of headache, it is important to keep in mind of such possibilities. About one-third of brain tumor patients complain of a headache as a primary symptom.

The intensity of pain, character and area of pain, and, especially, period of headache and accompanying neurologic symptoms can give an idea of investigating the intrinsic factors. Sudden onset of severe headache is related to intracranial disorders such as subarachnoid hemorrhage or meningitis rather than chronic headache. Headache due to sleep disorder, exertional headache, and paroxysmal headache in older adults also may imply intrinsic structural lesions such as lethargy, visual or limb symptoms, convulsion, and change in the state of mentality like in the case of headache accompanied by neurologic symptoms. Although chronic headache is commonly caused by migraine, tension, or depression, it may be related to intracranial lesions, head injury, cervical spondylosis, dental or ocular disease, temporomandibular 


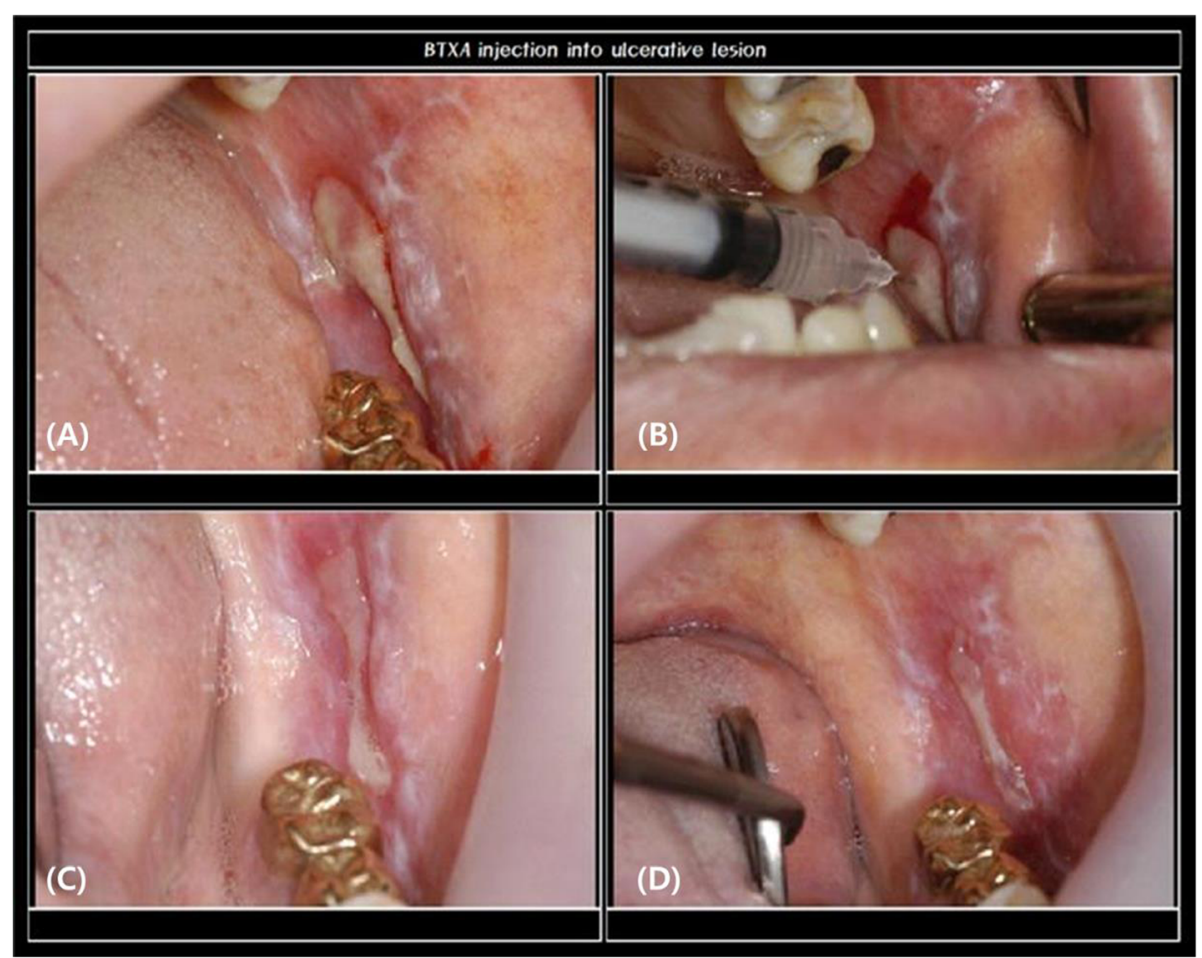

Fig. 8 A case with oral ulcerative lesion injected with botulinum toxin. a Clinical intraoral photograph at first visit. b Botulinum toxin injected 0.5 unit. c 2 weeks after injection. $\mathbf{d} 3$ weeks after injection

function disorder, sinusitis, hypertension, and various internal medicine disorders. Computed tomography $(\mathrm{CT})$ or magnetic resonance imaging (MRI) of head, electroencephalography, and lumbar puncture test is performed based on the initial diagnosis and patient's situation. You should diagnose and treat the primary neurologic disorder associated with headache with respect to the abovementioned diseases. However, very often, it is difficult to identify the source of pain in the case of chronic pain including referred pain and muscular disorders. We use various treatment protocols in managing chronic headache; removal of the intracranial and extracranial source is the principal method of approach. If the chronic headache persists even after the source has been removed, consider administration of drug (narcotic, non-narcotic analgesic), correction of occlusion, splint therapy, and relaxation of muscles by flaccid paralysis using botulinum toxin [21-26].

First and foremost, differential diagnosis is most important before proceeding with the treatment of chronic headache. Botulinum toxin treatment is preceded by removal of the source of pain depending on the differential diagnosis [21].

\section{Differential diagnosis}

A lot of associations have published the system which practically classifies craniofacial pain. Particularly, classification of chronic pain (1986) of the International
Association for the Study of Pain, classification of headache (1962) of the American Association for the Study of Headaches, and classification of headache (1994) of the American Association of Oral and Maxillofacial Surgery have a slight difference in the system [27, 28]. However, all of them use 8 systems of classification in common . Briefly, the pain can be classified into extracranial, intracranial, muscular, joint, neurological, causalgic, vascular, psychiatric (Table 1 ). We are going to make a differential diagnosis of head and neck pains based on these systems and discuss the possibilities of the use of botulinum toxin. The following are the classification and respective source of pain: extracranial structure disorder (teeth and craniofacial organs), intracranial (structures associated with the brain), muscular disorder (muscle, tendon, and connective tissue), joint disorder (bone, ligament, joint), neurologic disorder (peripheral nervous system), causalgic disorder (autonomous nervous system), vascular disorder (a vascular system), and psychiatric disorder (mental function). It is important to inspect these causes beforehand, and also diagnose diseases treatable with botulinum toxin injection.

There is a limit to using botulinum toxin to treat all patients with a chronic headache. You can make a differential diagnosis based on the above classification system, and it is a basic step to start with. 
Table 1 Eight practical classifications of headache occurring at cranio-maxillofacial area

\begin{tabular}{ll}
\hline Differential diagnosis groups & Cause of pain \\
\hline Extracranial disorder & Dental and craniofacial organ \\
Intracranial disorder & Structures associated with the brain \\
Muscular disorder & Muscle, tendon, connective tissue \\
Joint disorder & Bone, ligament, joint \\
Neuralgic disorder & Peripheral nervous system \\
Causalgia disorder & Autonomous nervous system \\
Vascular disorder & Vascular system \\
Psychiatric disorder & Mental disorder
\end{tabular}

The eight classifications useful in botulinum toxin treatment such as muscular disorder, joint disorder, neuralgic disorder, and causalgic disorder are reported in the literature. Borodic GE et al (2001) had reported that they observed satisfactory effect in 2 out of 3 patients after the treatment whose chief complaint was temporomandibular disorder symptoms and muscular disorder symptoms. Because most of the temporomandibular joint pain was due to masticatory muscles, Borodic GE et al. considered that the toxin was effective for such type of pain [21]. Since most of the muscular disorder, joint disorder, neuralgic disorder, causalgic disorder, and vascular disorder are accompanied by pain due to contraction of muscles, we think it is possible to use botulinum toxin in a wider range of treatment field.

Myofascial pain syndrome, myositis, fibrous myalgia, rigidity, and recurrent spasm are the typical diseases of headache caused by muscular disorders. These muscular disorders have typical trigger points in the muscle, so they can be used as a typical landmark while injecting botulinum toxin [25].

In general, symptoms of the neuralgic disorder are divided into paroxysmal and continuous; and an example for paroxysmal symptoms is trigeminal neuralgia (Figs. 9 and 10), and an example for continuous symptoms is post-herpetic pain (Fig. 11). Typical migraine, cluster headache, and temporal arteritis are examples of diseases representing vascular disorders.

\section{Mechanism of botulinum toxin for cranio-maxillofacial pain}

Botulinum toxin is shown to be effective for related pain diseases mainly associated with a muscular disease. Particularly, botulinum toxin is known to be effective in diseases with angialgia when a muscular disease such as tension headache or muscular disease occurs in combination with the temporomandibular joint disorder. Mechanism of botulinum toxin treatment is not well known; however, some of the literature reported about the mechanism [21-26, 29].

While botulinum toxin mainly acts on the $\alpha$-motor neuron, it is also known to act on the $\gamma$-motor neuron within the muscle spindles to reduce resting tone. Meanwhile, in animal testing, some researchers select a hypothesis stating that botulinum toxin has an anti-inflammatory effect, which is due to the effect of toxin suppressing the neurogenetic inflammation during headache treatment. However, more research

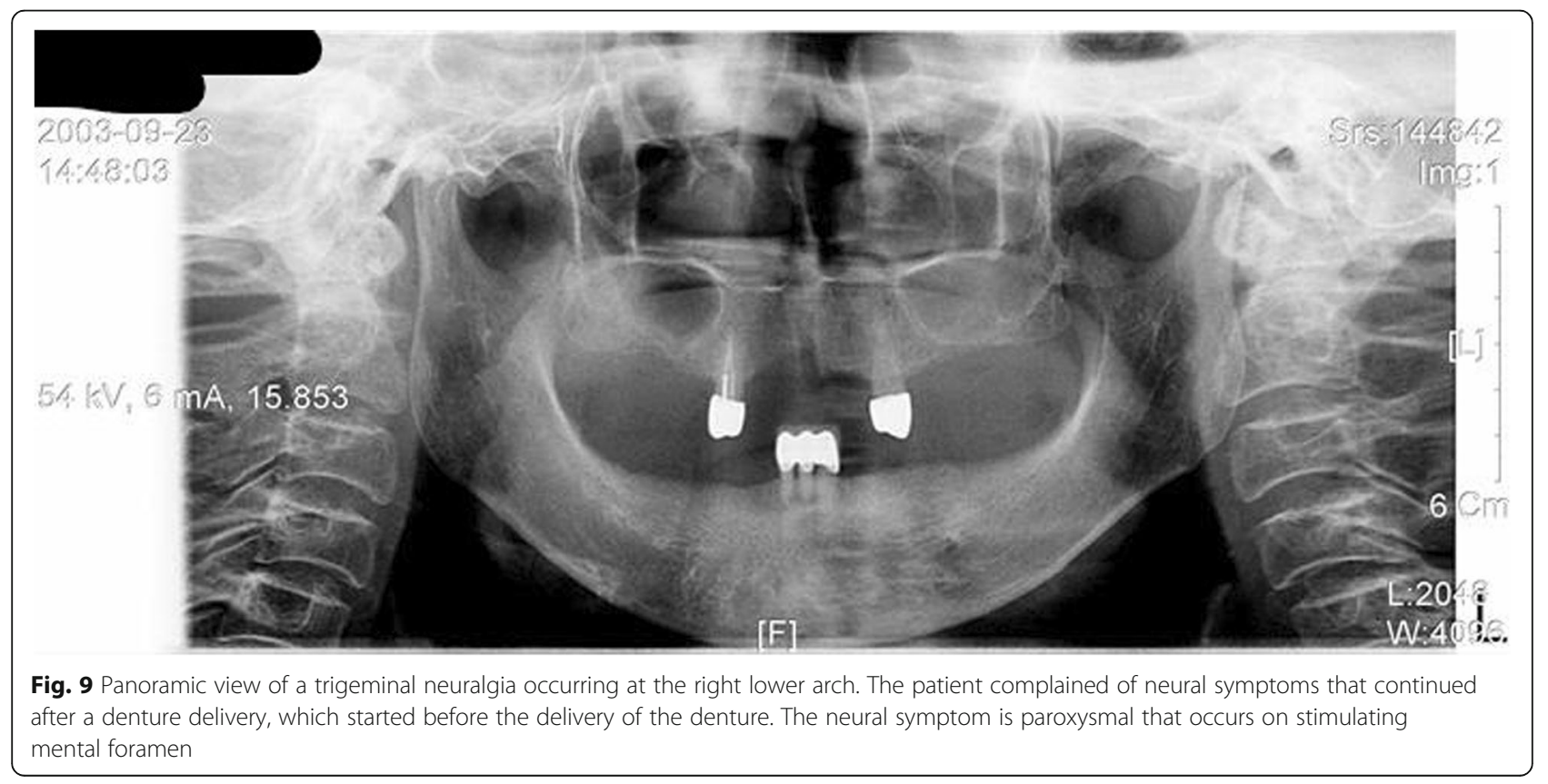




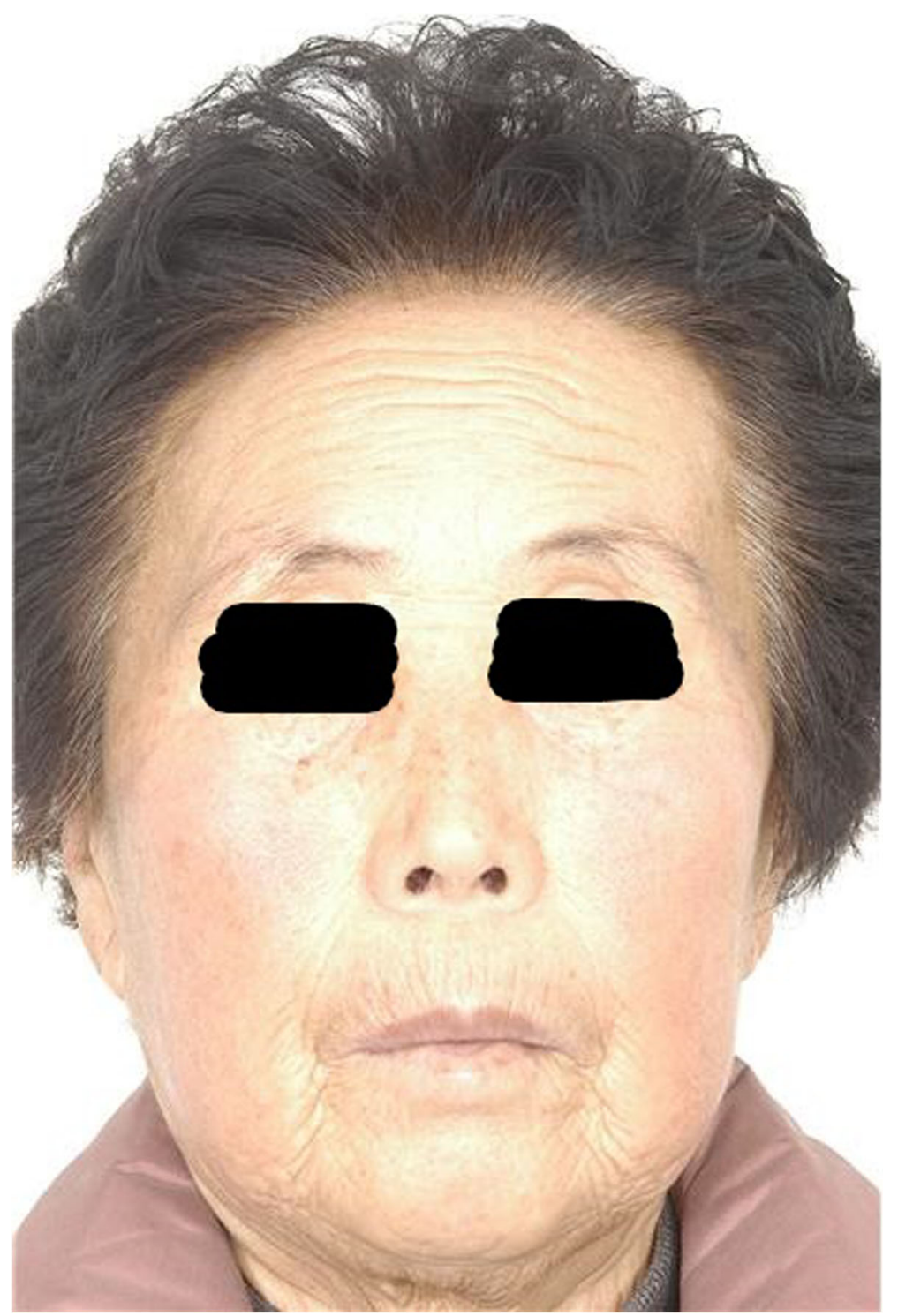

Fig. 10 As the trigeminal neuralgia is triggered from the mental foramen area, the patient showed significant improvement when she received botulinum toxin injection around the nerves. Unfortunately, symptoms recurred after a certain period of time

is required to investigate the exact mechanism of toxin in relieving pain. Apart from relieving pain, botulinum toxin is also known to be useful in certain areas in the dentistry field and effective for disorders such as bruxism and clenching. This therapeutic effect of botulinum toxin is known to be due to the removal of pain caused by headache or referred pain due to hypertonicity via relieving the contraction of the masseter and temporal muscles and reducing occlusal force [21]. According to Freund's 1999 study, the effect directly acting on the myoneural junction and the analgesic effect acting on the face and neck are all expected to play a role [30]. Some of the papers have suggested that the increase in vascularity due to muscle relaxation is more effective in eliminating trigger points or related pain than the analgesic effect $[23,25]$.

Similarly, if occurrence of habitual dislocation is suspected to be due to hypertonicity and disharmony in the lateral pterygoid muscle, injecting botulinum toxin into the lateral pterygoid muscle is known to alleviate habitual dislocation symptoms for a certain period of time by recovering muscular disharmony [22].

\section{Treatment of cranio-maxillo-facial pain using botulinum toxin}

According to Binder et al., when they injected various doses to migraine patients, they found out that 


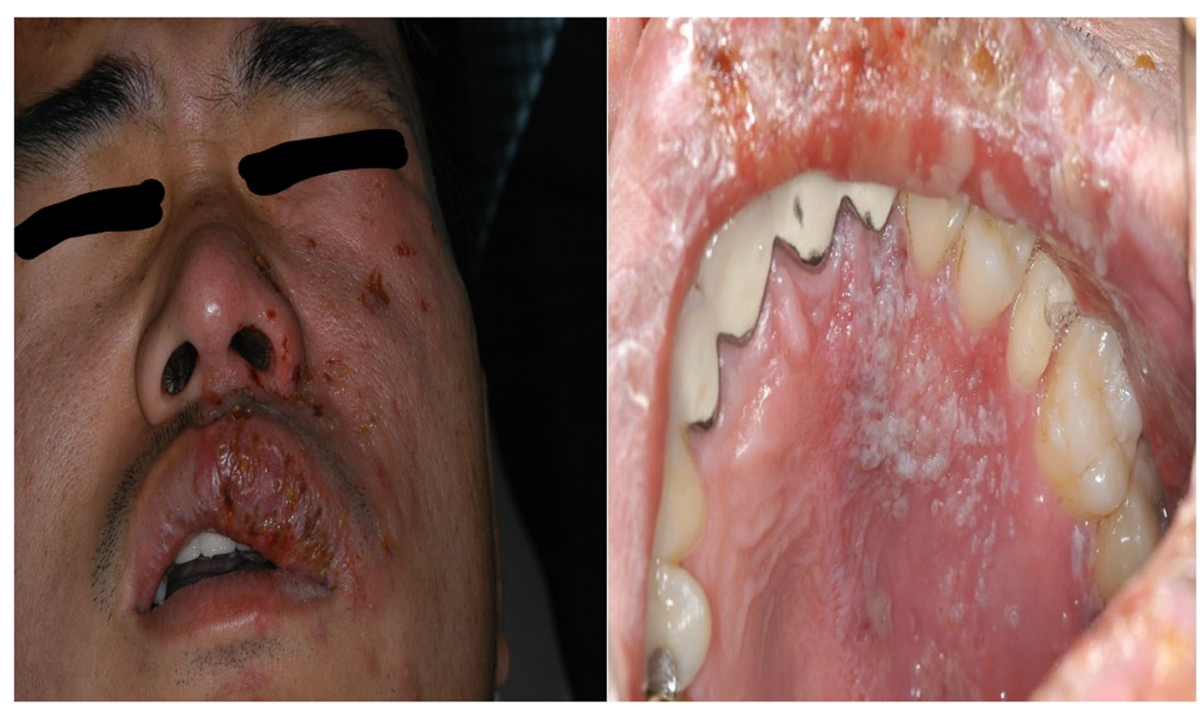

Fig. 11 Patient complained of severe pain in the left midface area due to herpes zoster. Symptoms subsided following constant antiviral injection. (Left) Extraoral photograph. (Right) Intraoral symptoms of herpes zoster. The symptoms are restricted to the left side of the mouth

botulinum toxin was effective in treating migraine [26, 29]. They reported that botulinum toxin was found to be effective when they injected the toxin into the trigger points where the tender area for stimulation exists in one of the skeletal muscles, tendons, and ligaments.

Generally, areas of injection for the treatment of headache are frontal, temporal, occipital, sternocleidomastoid, and glabellar. You should inject symmetrically, and remember not to exceed 5BU (Botox , BTXA ) per injection point. It is crucial not to exceed a dose of $25 \mathrm{BU}$ for each major muscle. Botulinum toxin is effective when injected into the trigger points of the major muscles known to cause headache including the frontal muscle, sternocleidomastoid muscle, and four masticatory muscles. Each point is shown in Fig. 12. Table 2 represents indication of botulinum toxin injection for headaches.

Contraindications for injecting into trigger zones are as follows: (1) Severe acute muscle injury, trauma, pain. (2) Patient allergic to the anesthetic agent. (3) Hemostatic disorder, bleeding tendency (diathesis), or patient taking an anticoagulant. (4) Occurrence of cellulitis in a trigger point.

Aseptic procedure and aspirating a needle are always critical while injecting botulinum toxin. Recognizing the location and shape of vessels passing through the muscles are important as well.

\section{Precaution of injection depending on the muscles Masseter muscle}

Approach extra-orally, inject into the trigger point of deep and superficial muscles with a $30 \mathrm{G}$ needle, watch out for facial artery. Similar to the case of square jaw patient as mentioned earlier, the injecting point is within a triangle formed by a line joining the mouth corner and tragus with the line connecting the angle of the mandible. Inject into the point $1.5 \mathrm{~cm}$ above the margin of the mandible and to the most bulging out portion of the muscle (Fig. 13).

\section{Temporal muscle}

Consider the location of the temporal artery and its branches; especially, it is advised to avoid injecting into the area near the tragus.

\section{Lateral pterygoid muscle}

With the patient's mouth slightly open, while palpating the sigmoid notch under the zygomatic bone, proceed the needle toward the opposite side of the face until you find the trigger point; proceed deep into the masseter muscle. You may proceed the needle until the tip of the needle is contacted with the lateral pterygoid plate. Withdraw the needle about $3-5 \mathrm{~mm}$ and inject into the lower head. Be careful not to inject into the maxillary artery and buccal nerve.

\section{Medial pterygoid muscle}

When taking the intraoral approach, enter medial to the pterygomandibular raphe, inject into the point posteriorly medial to the injection point used in local anesthesia. You can find the trigger point by moving the needle upward and downward.

\section{Sternocleidomastoid muscle}

Ask the patient to take a comfortable position so that the muscles do not contract, hold the muscles, and inject into the actual trigger point. Hold the entire 


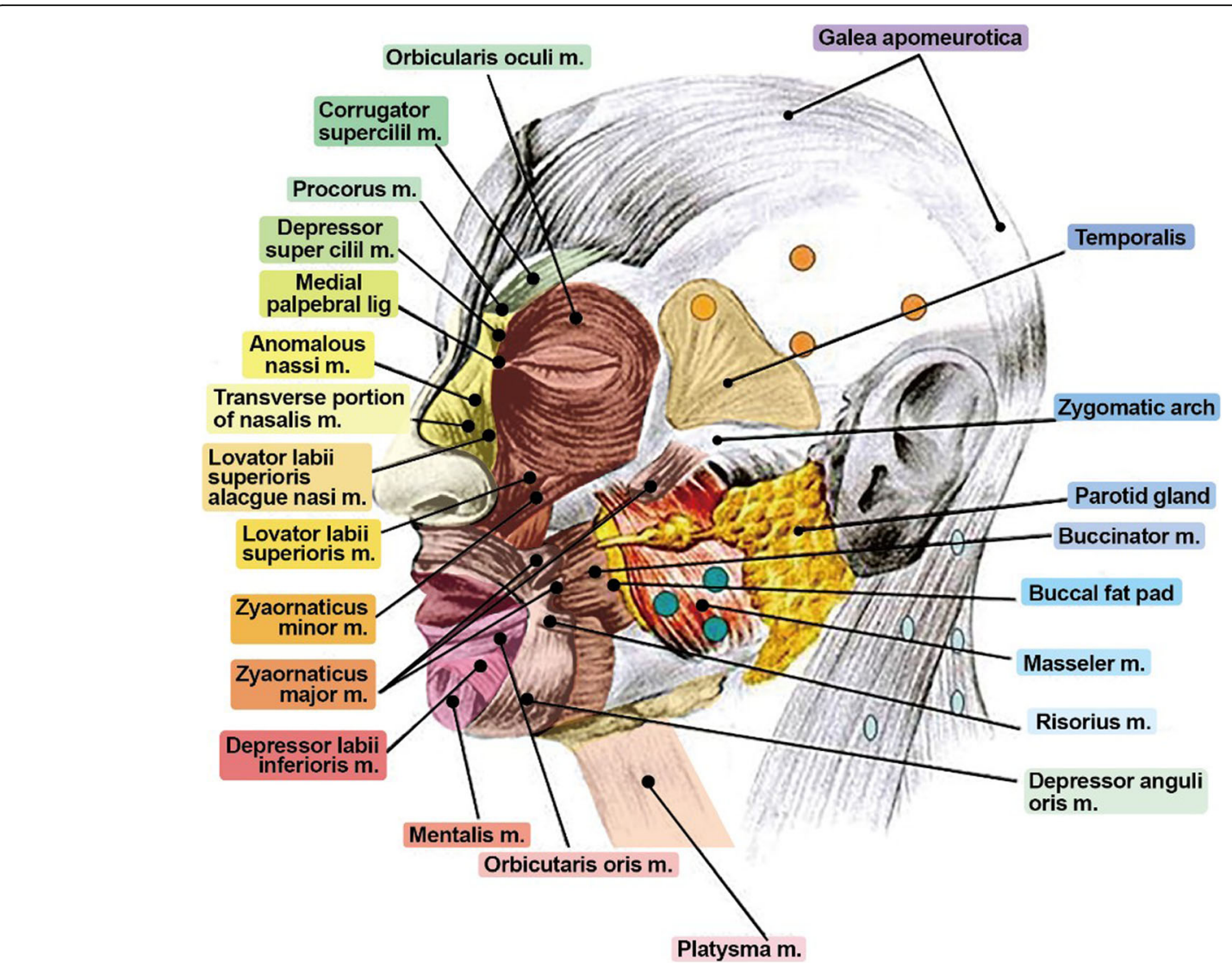

Fig. 12 Injection points of individual muscles. The principle is to inject 5BU per injection point

portion of the muscles with the hand to prevent injuring the carotid sheath and adjacent structures within the anterior cervical triangle. Be careful not to induce pneumothorax by perforating the lungs.

\section{Trapezius}

Be careful not to induce pneumothorax by perforating the lungs. Bypassing the shoulders, inject the toxin into the trigger point.

Table 2 Candidates for botulinum toxin A therapy for headaches

\footnotetext{
1. A case that is not resolved with any other treatment

2. Patient experiencing side effects from other treatment

3. Contraindicated for the patient who is undergoing a standard preventive program

4. Patient working in certain stressful environment

5. Patient misusing or abusing drugs

6. Patient who experienced muscle cramps in head and neck region

7. Patient who prefers botulinum toxin injection

8. Patient does not respond well to the standard treatment
}

It is recommended to inject $5 \mathrm{BU}$ per point for each muscle. In addition to the injection of botulinum toxin causing flaccid paralysis to such muscles, a combination of stretching therapy (passive, active elongation of muscles) will guarantee faster healing. If there is occlusion disharmony, consider using occlusal stabilization appliances and temporary administration of anti-inflammatory analgesics.

\section{Case report: Treatment of myofascial pain using botulinum toxin injection}

A 49-year-old man complained of headache with muscular fatigue in the morning caused by persistent bruxism. The patient was referred to our department because the patient had a temporary crown and bridge fractured that he received a week ago (Figs. 14 and 15). The patient had widespread trigger points in the sternocleidomastoid muscle and temporal muscle due to severe dystonia and persistent muscle rigidity. There was a significant improvement in the severity of bruxism and migraine after a month following injection of 20BU bilaterally into the masseter, temporal, and SCM. The patient's 


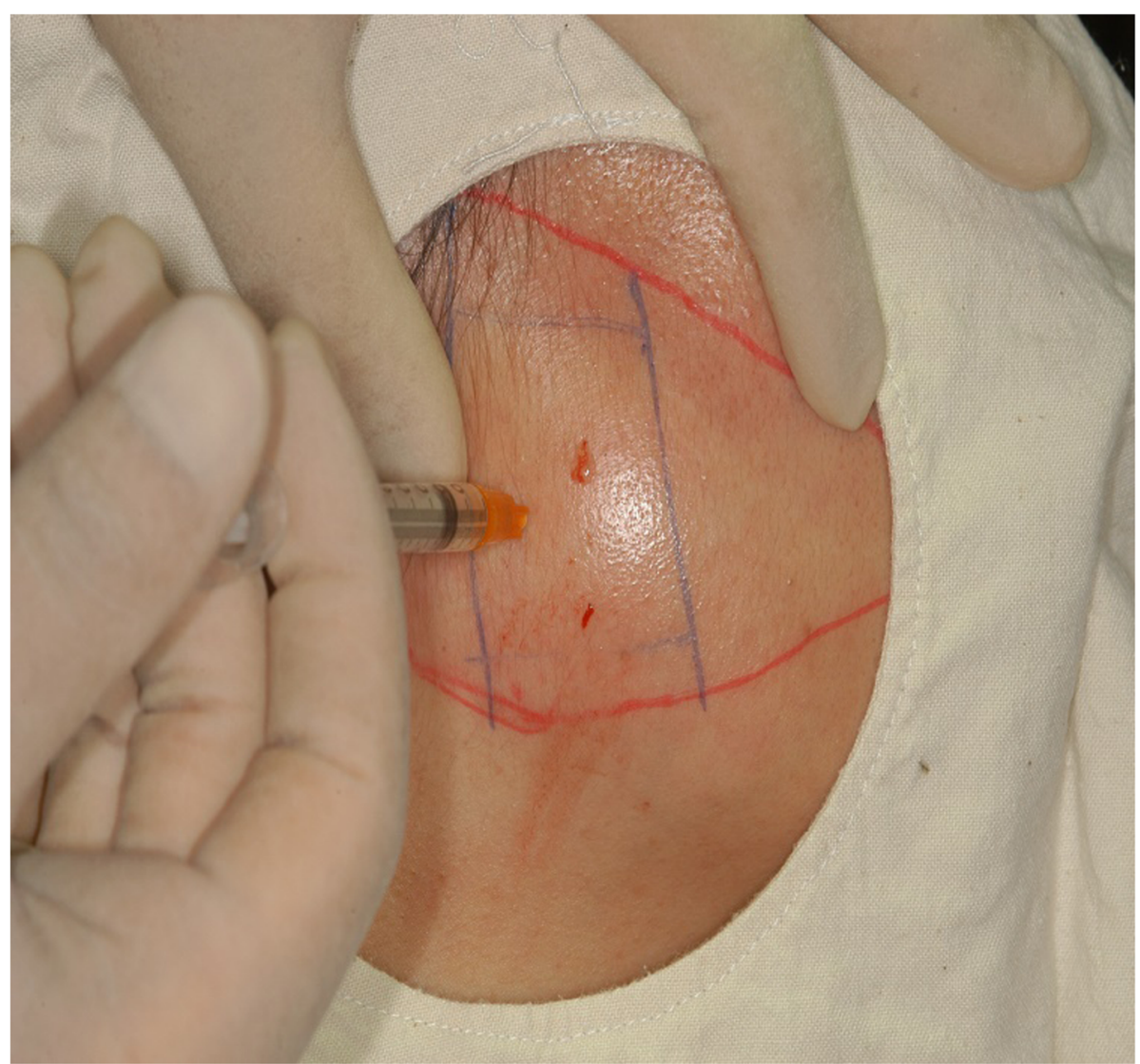

Fig. 13 Injecting botulinum toxin A with an insulin syringe into the injecting point of the masseter muscle within the triangle

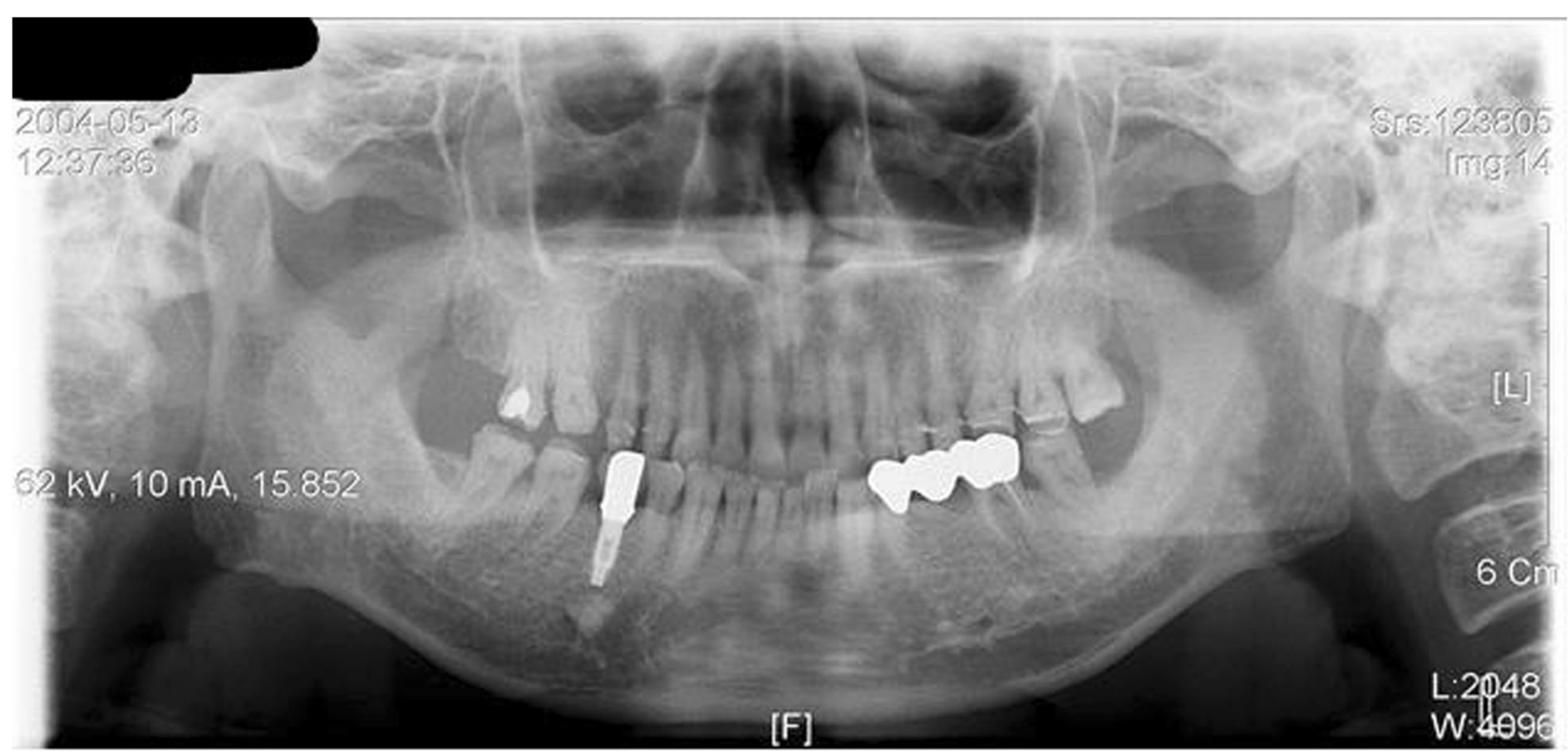

Fig. 14 A patient who had temporary crown and bridge in the upper and lower arches complained of chronic headache and muscular fatigue due to persistent bruxism. The patient has been transferred from the prosthodontic department to our department for treatment of chronic headache, relief of bruxism symptoms, and reduction of occlusal force 


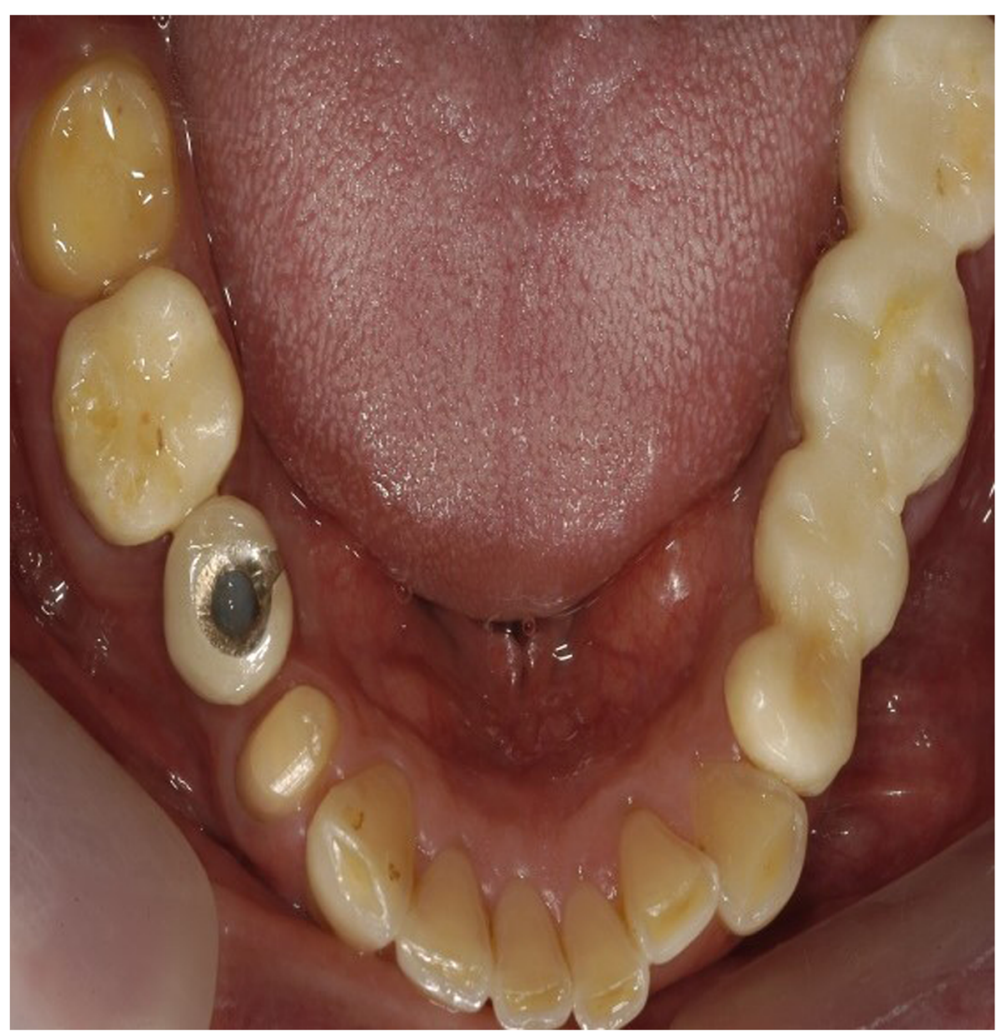

Fig. 15 One month after the initial visit. Patient's temporary crown remained intact and excessive occlusion was reduced

temporary crown remained intact, and he did not complain about the headache anymore thereafter.

\section{Contraindication and side effects of botulinum toxin}

Botulinum toxin treatment has less contraindication and side effects when compared with other general procedures. Ironically, problems arising from miscommunications between a patient and surgeon are the real problem. Therefore, it is always better for the surgeon to clarify with the patient beforehand if at all there is anything that the patient could miscomprehend [31-33]

For a 70-kg adult, administration of about 30 bottles of botox or BTXA at once is considered lethal. But according to the manufacturer's instruction, administration of a maximum 5 bottles ( $500 \mathrm{U}$ ) may cause botulism. Compliance with the standard dose regimen would generally prevent the occurrence of such problems.

\section{Contraindication}

\section{Patient with mental illness}

It is important to recognize a patient who has realistic expectations at the time of consultation. For the case of cosmetic purposes, consulting the patient before the procedure is of utmost important because it could eliminate factors capable of causing problems after the procedure.

\section{Patient with muscular disease}

The use of botulinum toxin is contraindicated in the patient with neuromuscular disease or myasthenia gravis.

\section{Patient taking drugs that show drug interaction}

These include aminoglycosides, penicillamine, quinine, calcium channel blocker, etc. They are known to enhance the action of botulinum toxin.

\section{Pregnancy and lactation}

There are no definite study results on this topic so far. But an animal study on rabbits has shown miscarriage and other side effects. Botulinum toxins are classified into Pregnancy category $\mathrm{C}$ drug, and there is a report that a woman who underwent botulinum toxin treatment gave normal birth to a child. There is no report of teratogenicity up to date; however, the use of botulinum toxin during pregnancy is generally not recommended.

\section{Side effects}

So far, the most common reason for using botulinum injection is to paralyze certain muscles, and the paralyzed muscle has a limit in its function. However, for a 
cosmetic procedure, there are other muscles to supplement the function, or the toxin is injected to the muscle that is not functionally important.

It is most important to discontinue drug intake that increases bleeding tendency such as aspirin or NSAIDs 7-10 days before injection to prevent postoperative bruising. Inject botulinum toxin into the area where the possibility of bleeding is as low as possible and pay close attention when injecting into the area around the eyes, or edge of the forehead. While pressing on to the injection area right after the injection often leads to bruising, it helps to subside the swelling [3, 4, 6, 32, 33].

\section{Conclusion}

Botulinum toxin is used in various ways such as temporarily resolving muscular problems in musculoskeletal temporomandibular disorders. It is used not only for treating these diseases but also for the cosmetic purpose to smoothen wrinkles caused by the muscle. The importance of knowledge on anatomical structures to reduce side effects is true.

\section{Abbreviations}

BU: botox unit: DU: dysport unitSMAS superficial musculoaponeurotic system

\section{Acknowledgements}

No acknowledgement.

\section{Authors' contributions}

SHY and DKK obtained the clinical case. KHK and KSS drafted the manuscript and critically revised the manuscript. All authors read and approved the final manuscript.

\section{Funding}

No funding source.

\section{Availability of data and materials}

Not applicable (data sharing not applicable to this article as no datasets were generated or analyzed during the current study).

\section{Ethics approval and consent to participate}

Not applicable.

\section{Consent for publication}

Written informed consent was obtained from the patient for the publication of this report and any accompanying images.

\section{Competing interests}

The authors declare that they have no competing interests.

Received: 14 June 2019 Accepted: 26 August 2019

Published online: 16 October 2019

\section{References}

1. Carruther A, Carruthers J (1998) Clinical indications and injections technique for the cosmetic use of botulinum A exotoxin. Dermatol Surg 24:1189-1194

2. Hankins CL, Strimling R, Roger GS (1998) Botulinum A toxin for glabellar wrinkles. Dose and response. Dermatol Surg 24:1181-1183

3. Bulstrode NW, Grobbelaar AO (2002) Long-term prospective follow-up of botulinum toxin treatment for facial rhytides. Aesthetic Plast Surg 26:356-359

4. Sarrabayrouse MA (2002) Indication and limitations for use of botulinum toxin for the treatment of facial wrinkles. Aesthetic Plast Surg 26:233-238

5. Jones OM, Brading AF, Mortensen NJ (2004) Mechanism of action of botulinum toxin on the internal anal sphincter. Br J Surg. 91:224-228
6. Yoon HC (2002) How to use botulinum toxin for treatment. Future Publishing, Busan

7. Wieder JM, Moy RL (1998) Understanding botulinum toxin: surgical anatomy of the frown, foreahead, and periocular region. Dermatol Surg 24:1172-1174

8. Edward Ellis III, Zide MF (1995) Surgical approaches to the facial skeleton. Wolters Kluwer, Philadelphia

9. Matarasso SL (1998) Complications of botulinum A exotoxin for hyperfunctional lines. Dermatol Surg. 24:1249-1254

10. Benedetto AV (1999) The cosmetic uses of botulinum toxin type A. Int J Dermatol. 38:641-655

11. Carruthers J, Carruthers A. (1991) Botulinum toxin use on glabellar wrinkles. Presented at the annual meeting of the American Society for dermatologic surgery Orlando Florida. 13-17 March. 1991.

12. Carruthers JD, Carruthers JA (1992) Treatment of glabellar frown lines with C. botulinum-A exotoxin. J Dermatol Surg Oncol 18:17-21

13. McCurdy JA Jr (1990) Cosmetic surgery of Asian face. Thieme Medical Publishers, New York

14. Price J, O'Day J (1993) A comparative study of tear secretion in blepharospasm and hemifacial spasm patients treated with botulinum toxin J Clin Neuroophthalmol 13:67-71.

15. Le Louarn C (2001) Botulinum toxin A and facial lines: the variable concentration. Aesthetic Plastic Surgery 25:73-84

16. Cheng CM, Chen JS, Patel RP (2006) Unlabeled uses of botulinum toxins: a review, part 1. Am J Health Syst Pharm 63:145-152

17. Cheng CM, Chen JS, Patel RP (2006) Unlabeled uses of botulinum toxins: a review, part 2. Am J Health Syst Pharm 63:225-232

18. Eisenberg E (2003) Diagnosis and treatment of recurrent aphthous stomatitis. Oral Maxillofac Surg Clin North Am 15:111-122

19. Yang TY, Jang TY (2009) The value of local botulinum toxin A injection in the treatment of the pain of aphthous ulcer. Eur Arch Otorhinolaryngol 266:445-448

20. Leviton A (1978) Epidemiology of headaches. In: Schoenberg BS (ed) Advances in Neurology. Raven Press, New York

21. Borodic GE, Acquadro M, Johnson EA (2001) Botulinum toxin therapy for pain and inflammatory disorders: mechanisms and therapeutic effects. Expert Opin Investig Drugs 10:1531-1544

22. Tan EK, Jankovic J (2000) Treating severe bruxism with botulinum toxin. J Am Dent Assoc 131:211-216

23. Kamanli A, Kaya A, Ardicoglu O, Ozgocmen S, Zengin FO, Bayik Y (2005) Comparison of lidocaine injection, botulinum toxin injection, and dry needling to trigger points in myofascial pain syndrome. Rheumatol Int 25:604-611

24. Wheeler AH, Goolkasian P, Gretz SS (2001) Botulinum toxin A for the treatment of chronic neck pain. Pain 94:255-260

25. Cheshire WP, Abashian SW, Mann JD (1994) Botulinum toxin in the treatment of myofascial pain syndrome. Pain 59:65-69

26. Binder WJ, Blitzer A (2003) Treatment of migraine headache with botulinum toxin type A. Facial Plast Surg Clin North Am 11:465-475

27. International Headache Society (1988) Classification and diagnostic criteria for headache disorders, cranial neuralgias and facial pain. Headache classification committee of the International Headache Society. Cephalalgia 7:1-96

28. Okeson JP (2008) The classification of orofacial pains. Oral Maxillofac Surg Clin North Am 20:133-144

29. Binder WJ, Brin MF, Blitzer A, Schoenrock LD, Pogoda JM (2000) Botulinum toxin type A (BOTOX) for treatment of migraine headaches: an open-label study. Otolaryngol Head Neck Surg 123:669-676

30. Freund B, Schwartz M, Symington JM (1999) The use of botulinum toxin for the treatment of temporomandibular disorders: preliminary findings. J Oral Maxillofac Surg 57:916-920

31. Archana MS (2016) Toxin yet not toxic: botulinum toxin in dentistry. Saudi Dent J 28:63-69

32. Lu DW, Lippitz J (2009) Complications of botulinum neurotoxin. Dis Mon 55:198-211

33. Niamtu J 3rd (2003) Botulinum toxin A: a review of 1,085 oral and maxillofacial patient treatments. J Oral Maxillofac Surg 61:317-324

\section{Publisher's Note}

Springer Nature remains neutral with regard to jurisdictional claims in published maps and institutional affiliations. 